\title{
Ovarian function after allogeneic hematopoietic stem cell transplantation in childhood and adolescence
}

\author{
A Vatanen 1,*, M Wilhelmsson ${ }^{2,3, *}$, B Borgström ${ }^{3,4}$, B Gustafsson ${ }^{3,4}$, M Taskinen ${ }^{1}$, \\ U M Saarinen-Pihkala ${ }^{1}$, J Winiarski ${ }^{3,4}$ and $K$ Jahnukainen ${ }^{1,2}$ \\ 'Division of Hematology-Oncology and Stem Cell Transplantation, Children's Hospital, Helsinki University Central \\ Hospital, University of Helsinki, FIN-00029 HUS Helsinki, Finland, Departments of ${ }^{2}$ Women's and Children's Health, \\ ${ }^{3}$ Pediatrics and ${ }^{4}$ Clinical Science, Innovation, and Technology, Karolinska Institutet, Karolinska University Hospital \\ Huddinge, Stockholm, Sweden \\ *(A Vatanen and M Wilhelmsson contributed equally to this work)
}

Correspondence should be addressed to A Vatanen

Email

anu.vatanen@hus.fi

\section{Abstract}

Objective: The aim of the study was to evaluate long-term ovarian function after allogeneic hematopoietic stem cell transplantation (HSCT) in childhood and adolescence.

Subjects and methods: Predictive factors for ovarian function were evaluated among 92 adult or pubertal female survivors transplanted at Huddinge and Helsinki University Hospital during 1978-2000, at a mean age of 9 \pm 4.3 years (range 1-19). At the time of the study a mean \pm s.D. of $13 \pm 5.5$ years (range 6-27) had elapsed since the HSCT and the mean age of the participants was $22 \pm 6.3$ years (range 9-41).

Results: Spontaneous puberty based on breast development occurred in 40 and menarche in 30 of the 70 girls who were prepubertal at transplantation. Six out of 20 girls who received HSCT after initiation of pubertal development recovered their ovarian function. Younger age at HSCT, conditioning without total body irradiation (TBI), and a non-leukemia diagnosis predicted the spontaneous menarche. The incidence of menarche was higher after fractioned vs single fraction TBI $(P<0.05)$, cyclophosphamide $(\mathrm{Cy})$ vs busulfan $(\mathrm{Bu})$-based conditioning $(P<0.05)$, and among leukemia patients transplanted at first remission vs later remissions $(P<0.01)$ and with no cranial irradiation (cranial radiotherapy, CRT) vs given CRT (14-24 Gy) $(P<0.01)$. The majority of recipients conditioned with only Cy vs TBI $(P<0.001)$ or vs Bu-based regimens $(P<0.01)$ showed preserved ovarian function and required no estrogen replacement at their latest follow-up visit at a mean age of $23 \pm 6.3$ years (range 15-41). Ten women became pregnant.

Conclusions: Patients conditioned with TBI or Bu-based regimes are at high risk of ovarian failure. Intensive antileukemia therapy before HSCT including CRT especially among relapsed patients may further decrease the possibility of spontaneous menarche.

\section{Introduction}

The ovary is particularly sensitive to the adverse effects of cancer treatment because of the finite number of germ cells present in the postnatal ovary $(1,2,3)$. Incomplete pubertal development has been reported to occur in $\sim 50 \%$ of prepubertal girls following hematopoietic stem cell transplantation (HSCT) $(4,5,6)$. The age at transplantation is critical; ovarian failure in adult women is usually irreversible, whereas in prepubertal girls, there is a better chance of a subsequent recovery and achievement of spontaneous menarche $(7,8,9)$. The risk of ovarian failure (c) 2014 European Society of Endocrinology Printed in Great Britain
Published by Bioscientifica Ltd. 
is dependent upon the conditioning regimen $(6,7,9,10)$. Total body irradiation (TBI), used in the preparative regimens for hematological malignancies, is deleterious to gonadal function $(5,6,7)$. Fractionation of the irradiation reduces the effect on the ovaries (7); girls treated with 12 Gy fractionated TBI (fTBI) are five times more likely to have a spontaneous recovery of the ovarian function than girls receiving single dose of TBI. The use of busulfan $(\mathrm{Bu})$ is also associated with a high incidence of gonadal failure $(9,11)$. Prepubertal patients conditioned with cyclophosphamide (Cy) for severe aplastic anemia (SAA) usually have a normal puberty (10).

The overall incidence of pregnancy following HSCT is known to be low $(<2 \%)$ with the exception of patients transplanted for SAA conditioned with Cy only (12-27\%) $(10,12)$. In general, studies indicate that fertility is most likely to be preserved in patients who undergo transplantation at young age and in those who receive non-TBIbased conditioning regimens.

Although data from retrospective cohort studies exist estimating the risk of pubertal problems and gonadal failure after HSCT, there are limited data comparing the reproductive risk of different conditioning regimens. There are also significant gaps in knowledge about females who will resume menstrual function after HSCT. In this retrospective cohort study, we evaluated signs of spontaneous puberty and menarche, need of estrogen substitution, and the outcome of conception in a cohort of 92 consecutive long-term survivors of allogeneic HSCT at childhood or adolescence at Huddinge and Helsinki University Hospitals. We describe here the natural history of ovarian dysfunction after conditioning regimens with and without TBI and provide information about premature menopause after HSCT in childhood and adolescence. A special strength of this study is the extended (median 13 years) longitudinal follow-up into puberty and adulthood of a defined cohort of stem cell transplanted girls and young women.

\section{Subjects and methods}

\section{Patients and follow-up program}

The female long-term survivors who were $<20$ years of age when receiving allogeneic HSCT at Karolinska University Hospital Huddinge, Stockholm, Sweden or Children's Hospital, Helsinki University Central Hospital, Helsinki, Finland, between 1978 and 2000 were identified through hospital records and enrolled in the study assessing longterm health outcome of childhood HSCT. The total number of female patients transplanted during this period was 104 in Huddinge and 80 in Helsinki. Among the total patient population, 102 females survived more than 5 years after HSCT. Out of these, 92 females (46 in Helsinki and 46 in Huddinge) had reached puberty or sexual maturity or showed ovarian failure (increased gonadotropin levels associated with failure to start or accomplish spontaneous puberty); by their latest follow-up visit, they had available follow-up hospital records and were enrolled in the study. Patient characteristics are listed in Table 1.

The follow-up program initially consisted of a semiannual and later annual contact at the outpatient clinic including complete late effect evaluation with clinical and

Table 1 Patient and transplant characteristics.

\begin{tabular}{|c|c|c|}
\hline & Study patients $n=92$ & $\%$ \\
\hline $\begin{array}{l}\text { Age at } \mathrm{HSCT} / \mathrm{mean} \pm \text { s.D. } \\
\text { (range) years }\end{array}$ & $9 \pm 4.3(1-19)$ & \\
\hline $\begin{array}{l}\text { Age at last visit/mean } \pm \text { s.D. } \\
\text { (range) years }\end{array}$ & $22 \pm 6.3(9-41)$ & \\
\hline $\begin{array}{l}\text { Follow-up time/mean } \pm \text { s.D. } \\
\text { (range) years }\end{array}$ & $13 \pm 5.5(6-27)$ & \\
\hline \multicolumn{3}{|l|}{ Pubertal stage at HSCT } \\
\hline Tanner 1 & 70 & 76 \\
\hline Tanner 2-3 & 12 & 13 \\
\hline Tanner 4-5 & 8 & 9 \\
\hline Unknown & 2 & 2 \\
\hline \multicolumn{3}{|l|}{ Diagnosis group } \\
\hline ALL & 33 & 36 \\
\hline AML & 24 & 26 \\
\hline SAA & 13 & 14 \\
\hline Others & 22 & 24 \\
\hline \multicolumn{3}{|l|}{ CRT for leukemia } \\
\hline No & 45 & \\
\hline Yes & 12 & \\
\hline \multicolumn{3}{|l|}{ Remission status of leukemia } \\
\hline CR 1 & 28 & \\
\hline CR 2-4 & 29 & \\
\hline \multicolumn{3}{|l|}{ Conditioning } \\
\hline sTBI+Cy & 29 & 32 \\
\hline $\mathrm{fTBI}+\mathrm{Cy}$ & 22 & 24 \\
\hline fTBI+Cy+ETO & 1 & 1 \\
\hline fTBI+ cytarabine & 16 & 17 \\
\hline fTBI+ melphalan & 3 & 3 \\
\hline $\mathrm{Bu}$ & 2 & 2 \\
\hline $\mathrm{Bu}+\mathrm{Cy}$ & 8 & 9 \\
\hline Cy only & 10 & 11 \\
\hline $\mathrm{Cy}+\mathrm{TNI}$ & 1 & 1 \\
\hline \multicolumn{3}{|l|}{ Donor } \\
\hline Related & 60 & 65 \\
\hline Unrelated & 32 & 35 \\
\hline \multicolumn{3}{|l|}{ Chronic GvHD } \\
\hline No & 66 & 72 \\
\hline Yes & 26 & 28 \\
\hline
\end{tabular}

$\mathrm{HSCT}$, hematopoietic stem cell transplantation; ALL, acute lymphoblastic leukemia; AML, acute myeloid leukemia; SAA, severe aplastic anemia; CRT, cranial radiotherapy; CR, complete remission; sTBI, single fraction total body irradiation; fTBI, fractioned total body irradiation; Cy, cyclophosphamide; ETO, etoposide; Bu, busulfan; TNI, total nodal irradiation; GvHD, graft vs host disease. 
laboratory examinations. The follow-up time comprised the time from HSCT to latest documented follow-up visit. Information about chronic graft vs host disease (GvHD), occurrence of signs of spontaneous puberty based on breast development, age at menarche, the use of estrogen substitution for non-contraceptive reasons, and pregnancies was collected from the follow-up records. Information about pubertal or postpubertal serum FSH levels before initiating the estrogen substitution, or any postpubertal value for those without estrogen substitution, was collected. Prepubertal serum FSH levels were excluded from the analysis. Serum FSH values of $1-12$ IU/1 were considered to be within the normal reference range. Persistently elevated levels of FSH (>25 IU/l) and absence of breast development were considered consistent with absence of spontaneous puberty. Premature menopause was documented by increased serum levels of FSH and failure to accomplish pubertal maturation or cessation of menstruation among girls who showed some ovarian activity after HSCT. Ovarian failure was documented by increased serum FSH levels, with no ovarian activity after HSCT.

Study patients were classified into three groups according to the treatment they had received before HSCT: i) leukemia group (acute myeloid leukemia (AML) $n=24$ and acute lymphoblastic leukemia (ALL) $n=33$ ); ii) patients with SAA $(n=13)$; and iii) others $(n=22)$; a heterogeneous group of patients with nonmalignant disorders or malignant diseases without cytotoxic therapy given before HSCT. Patients were further divided into three groups according to their pubertal status at HSCT: i) prepubertal (Tanner 1); ii) midpubertal (Tanner 2-3); and iii) latepubertal/postpubertal (Tanner 4-5). The Research Ethics Committees of Helsinki University Hospital and Karolinska University Hospital Huddinge approved the study.

\section{Treatment characteristics}

Treatment characteristics were collected from the hospital records. All leukemia patients were treated according to the consecutive Nordic protocols for ALL (since 1982) and AML (since 1984) $(13,14)$. Cy $120 \mathrm{mg} / \mathrm{kg}$ of body weight combined with 10 Gy single-fraction TBI (sTBI) was used as the standard conditioning for leukemia patients at the Karolinska University Hospital Huddinge during the years 1978-1995. fTBI at $12 \mathrm{~Gy}(3 \mathrm{~Gy} \times 4)$ was introduced in 1993. For patients with higher risk of leukemia relapse after HSCT, due to detectable pretransplant minimal residual disease or Philadelphia chromosome-positive ALL, etoposide $\left(900 \mathrm{mg} / \mathrm{m}^{2}\right)$ was added to the preparative regimen. The patients with AML transplanted from 1996 onwards have been conditioned with Cy $(120 \mathrm{mg} / \mathrm{kg})$ combined with $\mathrm{Bu}(16 \mathrm{mg} / \mathrm{kg})$. Patients with SAA received Cy $(200 \mathrm{mg} / \mathrm{kg})$. Patients with congenital metabolic defects received $\mathrm{Bu}(16 \mathrm{mg} / \mathrm{kg})$ usually with $\mathrm{Cy}$ (200 mg/kg). The Bu dosage was adjusted to reach the target area under the concentration-time curve.

Patients treated at the Helsinki University Hospital received sTBI (10-12 Gy) during the years 1978-1983 and fTBI (10-12 Gy) in 5-6 fractions since 1984, together with Cy $(120 \mathrm{mg} / \mathrm{kg})$, cytarabine $\left(36 \mathrm{~g} / \mathrm{m}^{2}\right)$ or melphalan $\left(210 \mathrm{mg} / \mathrm{m}^{2}\right)$. The total dose of $\mathrm{Bu}$ in all Bu-based regimens was $16 \mathrm{mg} / \mathrm{kg}$. Patients with SAA received Cy $(200 \mathrm{mg} / \mathrm{kg})$ with or without total lymph node irradiation (TNI) of $6 \mathrm{~Gy}$ or fTBI of $10 \mathrm{~Gy}$.

The number of study patients with different conditioning regimens is presented in Table 1 . The patients were further classified according to the conditioning characteristics into four groups: i) TBI-based conditioning (TBI), $n=71$; ii) Bu-based conditioning (Bu) without TBI or TNI, $n=10$; iii) conditioning with only Cy (Cy), $n=10$; and iv) conditioning with chemotherapy and TNI without TBI (TNI), $n=1$.

\section{Statistical analysis}

All data are presented as mean \pm s.D. and range. Statistical analyses were performed using the SPSS statistical software, version 20. Mann-Whitney $U$ test (for continuous variables), Fisher's exact test, and $\chi^{2}$ test (for categorical variables) were conducted to test differences between the groups. A $P$ value of $\leq 0.05$ indicated statistical significance. Odds ratios (ORs) and $95 \%$ CIs were calculated using bi- and multivariate logistic regression analyses. Categorical predictors used in logistic bi- and multivariate regression analysis were the dummy variables $(0 / 1)$ such as prepubertal at HSCT, leukemia diagnosis, SAA diagnosis, TBI, and single or fractioned TBI. The continuous variables were age at HSCT, follow-up time, serum level of FSH, and, for the leukemia group only, remission status at HSCT and cranial radiotherapy (CRT). One patient who received total nodal irradiation as the only form of irradiation was excluded from the analyses comparing the conditioning therapy.

\section{Results}

\section{Pubertal development and spontaneous menarche}

Altogether 70 girls were prepubertal at transplantation. Out of these, 40 (57\%) showed spontaneous onset of pubertal 
Table 2 Natural history of ovarian dysfunction after HSCT, defined by number of survivors with different pubertal status at the time of HSCT, by information about spontaneous onset of puberty and menarche, and by need of estrogen substitution at the latest follow-up visit.

\begin{tabular}{|c|c|c|c|c|c|c|c|c|c|c|c|c|c|c|c|}
\hline \multirow{4}{*}{$\begin{array}{l}\text { Spontaneous puberty post } \\
\text { HSCT }\end{array}$} & \multicolumn{15}{|c|}{ Pubertal status (total $n=92$ ) } \\
\hline & \multicolumn{8}{|c|}{ Prepubertal $(n=70)$} & \multicolumn{4}{|c|}{ Midpubertal $(n=12)$} & \multicolumn{2}{|c|}{$\begin{array}{l}\text { Postpubertal } \\
(n=8)\end{array}$} & \multirow{2}{*}{$\begin{array}{c}\text { UA } \\
(n=2)\end{array}$} \\
\hline & No & & Yes & & & & & & NA & & & & NA & & \\
\hline & 30 & & 40 & & & & & & & & & & & & 2 \\
\hline Spontaneous menarche post & No & & Yes & & & No & UA & & Yes & & No & UA & NA & & UA \\
\hline HSCT & 30 & & 30 & & & 7 & 3 & & 3 & & 3 & 6 & & & 2 \\
\hline Estrogen substitution at the & Yes & No & Yes & No & UA & Yes & No & UA & Yes & No & Yes & Yes & Yes & No & No \\
\hline latest follow-up visit & 27 & 3 & 7 & 16 & 7 & 7 & 1 & 2 & 2 & 1 & 3 & 6 & 3 & 5 & 2 \\
\hline
\end{tabular}

NA, not appicable; UA, information unavailable; HSCT, hematopoietic stem cell transplantation.

development and 30 (45\%) had spontaneous menarche (Table 2). Three mid-pubertal girls at HSCT had spontaneous menarche. The age for spontaneous puberty was $12.0 \pm 1.7$ years (range 9-16) and for spontaneous menarche $13 \pm 1.7$ years (range 9-17). Thirty girls needed hormonal induction of pubertal development and showed no spontaneous menarche. In multivariate logistics analysis, the only factor that significantly predicted spontaneous puberty was the age at HSCT (Table 3). For each increase of age of 1 year at the time of HSCT, the patient was 1.2 times more likely to not have a spontaneous puberty.

All girls who received only Cy $(200 \mathrm{mg} / \mathrm{kg})$ as conditioning experienced spontaneous puberty and menarche (Fig. 1). There was no difference in the incidence of spontaneous puberty or menarche among girls who received TBI compared with Bu-based conditioning (Fig. 1). In bivariate logistic analysis, TBI (vs no TBI) and leukemia diagnosis (vs no leukemia diagnosis) significantly decreased the probability of spontaneous menarche (Table 3). In multivariate step-wise analysis, TBI was the only significant predictor of no spontaneous menarche (Table 3). Chronic GvHD did not influence spontaneous puberty or menarche (Fig. 1).

Patients with a diagnosis of other than SAA had a six times higher probability to have no spontaneous menarche (Table 3). Patients with leukemia showed lower incidence of spontaneous menarche compared with patients with SAA (Fig. 1). Leukemia survivors with previous CRT and those transplanted in $\geq 2$ nd remission had lower incidence of spontaneous menarche than those with no CRT and those transplanted in the first remission (Fig. 1). Ten prepubertal leukemia survivors had received CRT with the dosage ranging from 14 to $24 \mathrm{~Gy}$ before HSCT: nine of them were transplanted in $\geq 2$ nd remission and four conditioned by sTBI and six by fTBI. Two of these girls had spontaneous puberty, but none of them had spontaneous menarche. Altogether, 22 prepubertal leukemia survivors had received transplantation in $\geq 2$ nd remission. Eight of them had spontaneous puberty, and only two had spontaneous menarche.

\section{Estrogen substitution at the latest follow-up visit}

Altogether 55 out of 83 (66\%) study patients with available treatment data received estrogen replacement therapy at their latest follow-up visit at a mean age of $23 \pm 6.3$ years (range 15-41) after a mean follow-up time of $14 \pm 6.0$ years (range 6-27) since the HSCT (Table 2). Forty-one of the $70(59 \%)$ girls who were prepubertal at transplantation

Table 3 Bivariate logistic regression analysis of risk factors for no spontaneous puberty or menarche and the need for estrogen substitution after allogeneic HSCT in childhood and adolescence.

\begin{tabular}{|c|c|c|c|}
\hline & \multicolumn{3}{|c|}{ Predictors } \\
\hline & OR & $95 \% \mathrm{Cl}$ & $P$ value \\
\hline \multicolumn{4}{|c|}{ No spontaneous puberty } \\
\hline Age at $\mathrm{HSCT}$ & 1.2 & $1.0-1.4$ & 0.015 \\
\hline \multicolumn{4}{|c|}{ No spontaneous menarche } \\
\hline No SAA diagnosis & 6.1 & $1.3-31.0$ & 0.030 \\
\hline TBI & 5.2 & $1.6-16.5$ & $0.006^{a}$ \\
\hline Leukemia diagnosis & 3.6 & $1.3-9.7$ & 0.011 \\
\hline Age at $\mathrm{HSCT}$ & 1.1 & $0.99-1.30$ & 0.06 \\
\hline $\mathrm{FSH}$ & 1.03 & $1.01-1.06$ & 0.002 \\
\hline LH & 1.09 & $1.03-1.14$ & 0.001 \\
\hline \multicolumn{4}{|c|}{ Estrogen substitution at the latest follow-up visit } \\
\hline TBls & 4.3 & $1.3-14.1$ & 0.016 \\
\hline SAA diagnosis & 0.2 & $0.1-0.9$ & 0.033 \\
\hline
\end{tabular}

HSCT, hematopoietic stem cell transplantation; SAA, severe aplastic anemia; TBI, total body irradiation.

${ }^{a}$ Result in multivariate analysis gives the same result. 

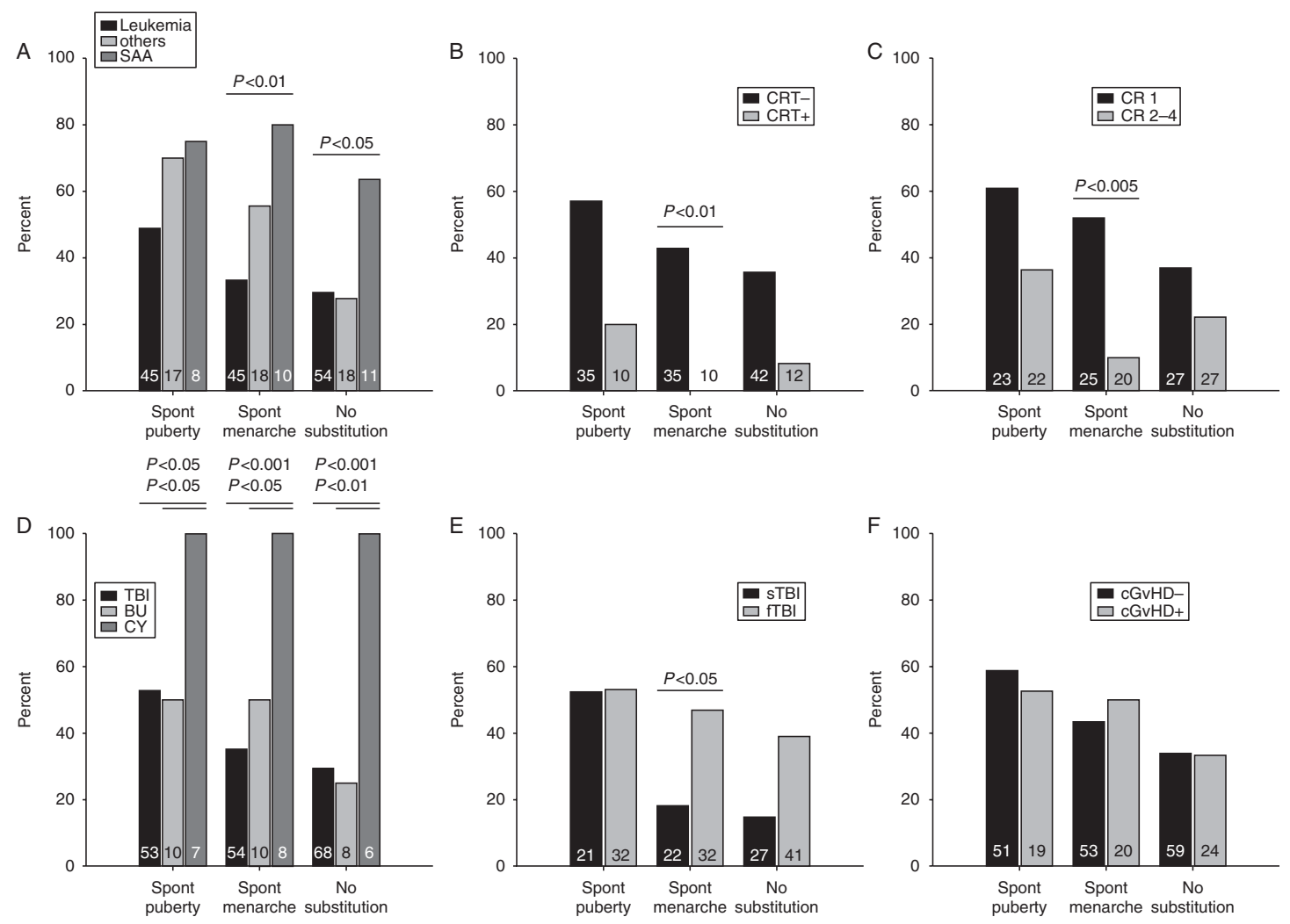

\section{Figure 1}

Incidence of spontaneous puberty, spontaneous menarche, and use of estrogen substitution at the latest follow-up visit according to $(A)$ primary diagnosis and $(B)$ use of cranial irradiation (CRT) before HSCT for leukemic patients.

(C) Remission status of leukemia at HSCT of leukemia patients; (D and E) conditioning therapy used; and (F) chronic graft vs

needed estrogen substitution at their latest follow-up visit. Twenty-seven of them showed no ovarian activity after HSCT and 14 initiated spontaneous puberty but entered into premature menopause at $11 \pm 4.4$ (range 6-21) years after HSCT, at a mean age of $17 \pm 3.1$ (range 13-25). Three midpubertal girls had documented spontaneous menarche after HSCT and two of them entered premature menopause at 5 and 13 years after HSCT, at the age of 18 and 22 years respectively. The survivors with premature menopause did not differ from the survivors with residual ovarian function in terms of age at HSCT, underlying disease, or type of conditioning treatment. Altogether 14 of the $20(70 \%)$ women who received HSCT after initiation of pubertal development received estrogen replacement therapy at their latest follow-up visit (Table 2). Six women recovered their menstrual function and needed no estrogen therapy (Table 2). None of the host (cGvHD) disease. Total numbers of patients included in the evaluations in each group and $P$ values are given. SAA, severe aplastic anemia; $C R$, complete remission; $T B I$, total body irradiation; $\mathrm{Bu}$, busulfan-based conditioning; $\mathrm{Cy}$, conditioning with only $\mathrm{Cy}$; fTBI, fractioned total body irradiation; sTBI, single fraction total body irradiation.

girls who received conditioning containing only $\mathrm{Cy}$ required estrogen replacement therapy. A higher proportion of patients conditioned with TBI $(P<0.001)$ or with $\mathrm{Bu}(P<0.01)$ needed estrogen replacement therapy compared with those conditioned with Cy (Fig. 1). No association to chronic GvHD was detected (Fig. 1). In bivariate logistic analysis, the only significant predictor for the use of estrogen substitution at the latest follow-up visit was conditioning with sTBI, and for no use of estrogen with the diagnosis of SAA (Table 3).

\section{Serum FSH levels}

Information about serum levels of FSH from the pubertal or postpubertal period was available for 76 survivors (Table 4). Serum FSH levels were measured $7 \pm 4.9$ years (range 0.1-21) after transplantation at the mean age of 
$15 \pm 3.7$ years (range 8-30). Measurements from the prepubertal period or after initiation of estrogen substitution were excluded. Significantly higher serum levels of FSH were detected among survivors who had received TBI or Bu-based conditioning compared with those with regimens containing only Cy (Table 4). No association to remission status of leukemia at HSCT, cranial irradiation (CRT) before HSCT, or chronic GvHD was detected. All 11 leukemia patients with CRT who had serum FSH measured showed serum FSH above the normal reference range and ten of them had serum FSH at menopausal level (>25 IU/1). In logistic regression analysis, the probability of spontaneous menarche was strongly correlated to serum levels of FSH. For each increase of FSH with 1 IU/l, the patient was 1.035 times more likely to not have spontaneous menarche.

\section{Fertility}

Ten women out of the 92 survivors had a total of 14 pregnancies and gave birth to 12 children (Table 5). Three of the children were born premature (gestational weeks 25 , 27 , and 32) and the others were born full-term. Four of the parenting women had leukemia and received fTBI.

Table 4 Pubertal and postpubertal serum FSH levels.

\begin{tabular}{|c|c|c|c|c|}
\hline & \multicolumn{4}{|c|}{ Serum FSH (IU/I) } \\
\hline & $n$ & Mean士s.D. & Range & $P$ value \\
\hline \multicolumn{5}{|c|}{ Pubertal stage at HSCT } \\
\hline Tanner 1 & 67 & $35 \pm 33.8$ & $1-143$ & \\
\hline Tanner 2-5 & 9 & $66 \pm 51.3$ & $2-160$ & \\
\hline \multicolumn{5}{|c|}{ Primary diagnosis } \\
\hline Leukemia & 48 & $43 \pm 36.5$ & $1-160$ & $<0.05^{\mathrm{a}}$ \\
\hline SAA & 8 & $15 \pm 12.4$ & $1-33$ & \\
\hline Others & 20 & $36 \pm 42.9$ & $2-143$ & \\
\hline \multicolumn{5}{|c|}{ CRT for leukemia } \\
\hline No & 37 & $42 \pm 40.3$ & $1-160$ & \\
\hline Yes & 11 & $46 \pm 20.1$ & 19-84 & \\
\hline \multicolumn{5}{|l|}{ Conditioning } \\
\hline TBI-based & 59 & $39 \pm 35.5$ & $1-160$ & \\
\hline Bu-based & 10 & $57 \pm 48.3$ & $3-143$ & $<0.05^{b}$ \\
\hline Cy-based & 7 & $7 \pm 8.0$ & $1-25$ & $<0.01^{b}$ \\
\hline \multicolumn{5}{|c|}{ Fractionation of TBI } \\
\hline fTBI & 34 & $39 \pm 39.7$ & $2-160$ & \\
\hline sTBI & 25 & $40 \pm 29.5$ & $1-101$ & \\
\hline \multicolumn{5}{|c|}{ Chronic GvHD } \\
\hline No & 55 & $42 \pm 38.6$ & $1-160$ & \\
\hline Yes & 21 & $30 \pm 33.1$ & $1-107$ & \\
\hline
\end{tabular}

HSCT, hematopoietic stem cell transplantation; SAA, severe aplastic anemia; CRT, cranial radiotherapy; $C R$, complete remission; TBI, total body irradiation; Bu, busulfan; Cy, cyclophosphamide; fTBI, fractioned TBI; STBI, single fraction TBI; GvHD, graft vs host disease.

${ }^{a}$ Compared with SAA.

${ }^{b}$ Compared with TBI. Serum FSH values 1-12 IU/I were considered within the normal reference range and serum values $>25 \mathrm{IU} / \mathrm{l}$ at menopausal level.
Six women had nonmalignant diagnoses and three of them had received Bu-based conditioning, two only $\mathrm{Cy}$, and one sTBI with ovarian shielding.

\section{Discussion}

This cohort study reports the natural history of ovarian dysfunction in 92 consecutive pubertal or adult female survivors after an allogeneic HSCT at childhood or adolescence at Huddinge or Helsinki University Hospitals. The novel aspect and strength of this study are the extended (median 13 years) longitudinal follow-up into puberty and adulthood of a well-defined cohort of stem cell transplanted girls and young women. Failing ovarian function associated significantly to age at HSCT, which confirms the earlier observations that very young patients with larger ovarian reserves have better chances, at least temporarily, to recover their ovarian function after HSCT $(7,8,9)$. Altogether, 41 out of 70 (59\%) prepubertal girls, 11 of 12 (92\%) midpubertal girls, and three of eight (38\%) postpubertal girls at HSCT needed sex hormone replacement therapy by their latest clinical visit. The majority of patients with estrogen substitution showed no spontaneous ovarian activity after HSCT, which suggests that they sustained ovarian failure at the time of HSCT. Altogether, 14 out of 40 prepubertal girls and two out of three midpubertal girls temporarily recovered their ovarian function after HSCT with spontaneous pubertal development or spontaneous menarche, but had a premature cessation of ovarian function at a very young age of 17 and 20 years respectively. The identification of this patient group will remain a major challenge for fertility counselling because ovarian recovery in these women is likely to be followed by a premature menopause. The period of recovery may be a window of opportunity to conceive naturally (12) or by assisted conception techniques (15) and the patients may benefit from fertility preservation techniques such as cryopreservation of mature oocytes. Only regular follow-up can identify this period of recovery.

Girls and young women conditioned with TBI or Bu-based regimens were shown to be at an equally high risk of declining ovarian function and premature menopause compared with patients conditioned with Cy only. TBI was especially damaging when given in a single fraction. The anti-leukemia therapy including CRT before HSCT or transplantation after disease relapse further decreased the possibility to retain ovarian function among leukemia patients. The use of CRT at high doses (>30 Gy) can potentially damage the 
Table 5 Patients with offspring.

\begin{tabular}{|c|c|c|}
\hline Patient no. & Diagnosis & Age at HSCT \\
\hline 1 & ALL & 15 \\
\hline 2 & ALL & 12 \\
\hline 3 & ALL & 10 \\
\hline 4 & ALL & 7 \\
\hline 5 & SCID & 10 \\
\hline 6 & $\mathrm{FHL}$ & 6 \\
\hline 7 & Thalassemia & 2 \\
\hline 8 & Mb Gaucher & 2 \\
\hline 9 & SAA & 15 \\
\hline 10 & SAA & 14 \\
\hline
\end{tabular}

\begin{tabular}{|c|c|}
\hline Pubertal stage at HSCT & Conditioning \\
\hline 3 & fTBI+Cy \\
\hline 1 & fTBI+ cytarabine \\
\hline 1 & fTBI+ cytarabine \\
\hline 1 & fTBI+ cytarabine \\
\hline Unknown & $\begin{array}{l}\text { sTBI+Cy+ovarian } \\
\text { shielding }\end{array}$ \\
\hline 1 & $\mathrm{Bu}$ \\
\hline 1 & $\mathrm{Bu}$ \\
\hline 1 & $\mathrm{Bu}+\mathrm{Cy}$ \\
\hline 3 & Cy \\
\hline Unknown & Cy \\
\hline
\end{tabular}

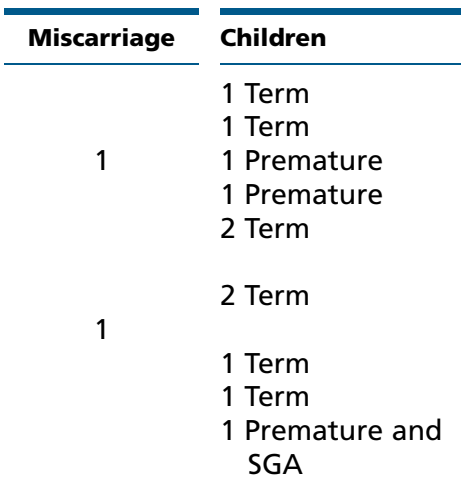

HSCT, hematopoietic stem cell transplantation; ALL, acute lymphoblastic leukemia; SCID, severe combined immunodeficiency; FHL, familial hematophagocytic lymphohistiocytosis; Mb, morbus; SAA, severe aplastic anemia; fTBI, fractioned total body irradiation; sTBI, single fraction total body irradiation; Bu, busulfan; Cy, cyclophosphamide; SGA, small for gestational age.

hypothalamic pituitary region and result in gonadotropin-releasing hormone deficiency (16). In this study, none of the patients treated with CRT (14-24 Gy) showed signs of hypogonadotrophic hypogonadism. Instead, serum levels of FSH were at menopausal level in ten of the 11 patients who received CRT. It is therefore more probable that intensive conventional chemotherapy that associated to CRT especially among relapsed patients (nine of ten prepubertal girls transplanted in $\geq 2$ nd remission had received CRT) caused direct ovarian damage and led to lack of spontaneous menarche.

Altogether ten females transplanted under the age of 20 became pregnant during the follow-up. Four women with leukemia conceived after fTBI and three women after Bu-based conditioning. One woman became pregnant after sTBI. Her ovaries were protected by shielding, which encourages us to believe that shielding potentially preserves fertility after TBI (17). Clearly, shielding is not always an option for patients with malignant diseases and novel fertility preservation methods need to be developed. If there is enough time for ovarian stimulation before initiation of gonadotoxic therapy, cryopreservation of mature oocytes for menstruating adolescent girls is currently the clinical option for parenting an own child $(18,19,20)$. If treatment cannot be delayed, cryopreservation of ovarian tissue offers a novel option for fertility preservation $(18,19,20)$ also for young sexually immature girls (20). Leukemia patients constitute an important group who could benefit from novel reimplantation techniques that have resulted in pregnancies and live births $(19,20)$. However, concern has been expressed that the method possibly carries a risk of leukemia relapse $(21,22)$.

This study is limited by our inability to establish the precise incidence of fertility following HSCT because the wish to become a parent or the waiting time for a desired pregnancy was not recorded. The mean age of 23 years at the latest hospital visit is also low for both menopause and fertility studies and may only display the beginning of the fertile period among the oldest group of transplanted girls. Accordingly, in this study, six out of ten women who became pregnant were older than 10 years at the time of HSCT. Another limitation was a low number of leukemia patients treated with non-TBI conditioning. This prevented us from evaluating the effects of previous CRT on ovarian function among non-TBI conditioned patients and from studying whether intensive conventional chemotherapy (patients transplanted in $\geq 2$ nd remission) played a major role in a lower probability of spontaneous menarche.

In conclusion, while patients conditioned with Cy are likely to regain their ovarian function, only $29 \%$ of those conditioned with TBI and 25\% of those with Bu-based regimens demonstrated long-term ovarian function and were without sex steroid replacement therapy at their latest follow-up visit. Failing ovarian function was more frequent among those who were older at HSCT and conditioned with TBI. The use of CRT for anti-leukemia therapy and transplantation in $\geq 2$ nd leukemia remission further decreased the possibility of spontaneous menarche. The majority of patients showed no ovarian activity after HSCT, suggesting that the entire follicle pool was depleted at HSCT. Some pre- and midpubertal survivors temporarily recovered their ovarian function but entered premature menopause at the ages of 17 and 20 years. For survivors with residual ovarian function, there will be prospects to conceive naturally and they may benefit from assisted conception techniques and fertility preservation after HSCT. 


\section{Declaration of interest}

The authors declare that there is no conflict of interest that could be perceived as prejudicing the impartiality of the research reported.

\section{Funding}

This work was supported by the Swedish Barncancerfonden, the Finnish Cancer Society, the Finnish Pediatric Research Foundation, Paulo Foundation, the Nona and Kullervo Väre Foundation, and the Academy of Finland.

\section{Acknowledgements}

We thank Björn Jonsson for performing the statistical analysis and Jaana Vettenranta, Satu Ranta, and Pirjo Valtonen for skilful technical assistance.

\section{References}

1 Faddy MJ, Gosden RG, Gougeon A, Richardson SJ \& Nelson JF. Accelerated disappearance of ovarian follicles in mid-life: implications for forecasting menopause. Human Reproduction 199210 1342-1346.

2 Tilly JL. Commuting the death sentence: how oocytes strive to survive. Nature Reviews. Molecular Cell Biology 20012 838-848. (doi:10.1038/ 35099086)

3 Wallace WH \& Kelsey TW. Human ovarian reserve from conception to the menopause. PLOS ONE 20105 e8772. (doi:10.1371/journal.pone. 0008772)

4 Sarafoglou K, Boulad F, Gillio A \& Sklar C. Gonadal function after bone marrow transplantation for acute leukemia during childhood. Journal of Pediatrics 1997130 210-216. (doi:10.1016/S0022-3476(97)70345-7)

5 Bakker B, Massa GG, Oostdijk W, Van Weel-Sipman MH, Vossen JM \& Wit JM. Pubertal development and growth after total-body irradiation and bone marrow transplantation for haematological malignancies. European Journal of Pediatrics 2000159 31-37. (doi:10.1007/ s004310050006)

6 Parsons SK, Phipps S, Sung L, Baker KS, Pulsipher MA \& Ness KK. NCI, NHLBI/PBMTC First International Conference on Late Effects after Pediatric Hematopoietic Cell Transplantation: health-related quality of life, functional, and neurocognitive outcomes. Biology of Blood and Marrow Transplantation 201218 162-171. (doi:10.1016/j.bbmt.2011.12.501)

7 Sanders JE, Buckner CD, Amos D, Levy W, Appelbaum FR, Doney K, Storb R, Sullivan KM, Witherspoon RP \& Thomas ED. Ovarian function following marrow transplantation for aplastic anemia or leukemia. Journal of Clinical Oncology 19886 813-818.

8 Spinelli S, Chiodi S, Bacigalupo A, Brasca A, Menada MV, Petti AR, Ravera G, Gualandi F, VanLint MT, Sessarego M et al. Ovarian recovery after total body irradiation and allogeneic bone marrow transplantation: long-term follow up of 79 females. Bone Marrow Transplantation 199414 373-380. (doi:10.1038/jcbfm.1994.48)

9 Borgmann-Staudt A, Rendtorff R, Reinmuth S, Hohmann C, Keil T, Schuster FR, Holter W, Ehlert K, Keslova P, Lawitschka A et al. Fertility after allogeneic haematopoietic stem cell transplantation in childhood and adolescence. Bone Marrow Transplantation 201247 271-276. (doi:10.1038/bmt.2011.78)

10 Sanders JE, Woolfrey AE, Carpenter PA, Storer BE, Hoffmeister PA, Deeg HJ, Flowers ME \& Storb RF. Late effects among pediatric patients followed for nearly 4 decades after transplantation for severe aplastic anemia. Blood 2011118 1421-1428. (doi:10.1182/blood-201102-334953)

11 Sanders JE, Hawley J, Levy W, Gooley T, Buckner CD, Deeg HJ, Doney K, Storb R, Sullivan K, Witherspoon R et al. Pregnancies following high-dose cyclophosphamide with or without high-dose busulfan or total-body irradiation and bone marrow transplantation. Blood $1996 \mathbf{8 7}$ 3045-3052.

12 Socié G, Salooja N, Cohen A, Rovelli A, Carreras E, Locasciulli A, Korthof E, Weis J, Levy V \& Tichelli A. Nonmalignant late effects after allogeneic stem cell transplantation. Blood $20031013373-3385$ (doi:10.1182/blood-2002-07-2231)

13 Gustafsson G, Schmiegelow K, Forestier E, Clausen N, Glomstein A, Jonmundsson G, Mellander L, Mäkipernaa A, Nygaard R \& SaarinenPihkala UM. Improving outcome through two decades in childhood ALL in the Nordic countries: the impact of high-dose methotrexate in the reduction of CNS irradiation. Nordic society of pediatric haematology and oncology (NOPHO). Leukemia 200014 2267-2275. (doi:10.1038/sj.leu.2401961)

14 Lie SO, Abrahamsson J, Clausen N, Forestier E, Hasle H, Hovi L, Jonmundsson G, Mellander L, Siimes MA, Yssing M et al. Long-term results in children with AML: NOPHO-AML Study Group - report of three consecutive trials. Leukemia 200519 2090-2100. (doi:10.1038/sj. leu.2403962)

15 Das M, Shehata F, Son WY, Tulandi T \& Holzer H. Ovarian reserve and response to IVF and in vitro maturation treatment following chemotherapy. Human Reproduction 201227 2509-2514. (doi:10.1093/ humrep/des143)

16 Cohen A, Békássy AN, Gaiero A, Faraci M, Zecca S, Tichelli A \& Dini G \& EBMT Paediatric and Late Effects Working Parties. Endocrinological late complications after hematopoietic SCT in children. Bone Marrow Transplantation 200841 S43-A48. (doi:10.1038/bmt.2008.54)

17 Nakagawa K, Kanda Y, Yamashita H, Hosoi Y, Oshima K, Ohtomo K, Ban N, Yamakawa S, Nakagawa S \& Chiba S. Preservation of ovarian function by ovarian shielding when undergoing total body irradiation for hematopoietic stem cell transplantation: a report of two successful cases. Bone Marrow Transplantation 200637 583-587. (doi:10.1038/sj. bmt.1705279)

18 Lee SJ, Schover LR, Partridge AH, Patrizio P, Wallace WH, Hagerty K, Beck LN, Brennan LV \& Oktay K. American Society of Clinical Oncology recommendations on fertility preservation in cancer patients. Journal of Clinical Oncology 200624 2917-2931. (doi:10.1200/ JCO.2006.06.5888)

19 ISFP Practice Committee, Kim SS, Donnez J, Barri P, Pellicer A, Patrizio P, Rosenwaks Z, Nagy P, Falcone T, Andersen C et al. Recommendations for fertility preservation in patients with lymphoma, leukemia, and breast cancer. Journal of Assisted Reproduction and Genetics 201229 465-468. (doi:10.1007/s10815-012-9786-y)

20 Jadoul P, Dolmans MM \& Donnez J. Fertility preservation in girls during childhood: is it feasible, efficient and safe and to whom should it be proposed? Human Reproduction Update 201016 617-630. (doi:10.1093/ humupd/dmq010)

21 Dolmans MM, Marinescu C, Saussoy P, Van Langendonckt A, Amorim C \& DonnezJ. Reimplantation of cryopreserved ovarian tissue from patients with acute lymphoblastic leukemia is potentially unsafe. Blood 2010116 2908-2914. (doi:10.1182/blood-2010-01-265751)

22 Jahnukainen K, Tinkanen H, Wikström A, Dunkel L, SaarinenPihkala UM, Mäkinen S, Asadi Azarbaijani B, Oskam IC \& Vettenranta K. Bone marrow remission status predicts leukemia contamination in ovarian biopsies collected for fertility preservation. Leukemia 201327 1183-1185. (doi:10.1038/leu.2012.279)

Received 22 August 2013

Revised version received 28 October 2013

Accepted 31 October 2013 